\title{
Outbreak of a beta-lactam resistant non- typeable Haemophilus influenzae sequence type 14 associated with severe clinical outcomes
}

Madelen Andersson ${ }^{1}$, Fredrik Resman ${ }^{2 *}$, Rickard Eitrem ${ }^{3}$, Peter Drobni ${ }^{4}$, Kristian Riesbeck ${ }^{2}$, Gunnar Kahlmeter ${ }^{4,5}$ and Martin Sundqvist ${ }^{4,6}$

\begin{abstract}
Background: During October 2011 several residents and staff members at a long-term care facility (LTCF) for elderly fell ill with respiratory symptoms. Several of the residents required hospitalization and one died. Non-typeable Haemophilus influenzae (NTHi) was identified as the causative pathogen.

Methods: A descriptive analysis of the outbreak and countermeasures was performed. For each identified bacterial isolate implied in the outbreak, standard laboratory resistance testing was performed, as well as molecular typing and phylogenetic analysis.

Results: The identified H. influenzae was beta-lactamase negative but had strikingly high MIC-values of ampicillin, cefuroxime and cefotaxime. All isolates displayed the same mutation in the ftsl gene encoding penicillin-binding protein (PBP) 3, and all but one were identified as sequence type 14 by Multilocus Sequence Typing (MLST). In total 15 individuals in connection to the LTCF; 8 residents, 6 staff members and one partner to a staff member were colonized with the strain.
\end{abstract}

Conclusion: This report illustrates the existence of non-typeable $H$. influenzae with high virulence, and furthermore emphasizes the importance of continuous surveillance of possible outbreaks in health care facilities and prompt measures when outbreaks occur.

Keywords: Ampicillin, Betalactamase negative, BLNAR, Long-term care facility, Haemophilus influenzae, Non-typeable, Outbreak, Resistant

\section{Background}

Haemophilus influenzae is a respiratory tract commensal that is associated with upper respiratory tract infections, but also meningitis and septicemia [1]. After the introduction of a $H$. influenzae type b (Hib) vaccine in the childhood vaccination program in 1992, non-typeable $H$. influenzae (NTHi) have dominated $H$. influenzae morbidity in Sweden [2], but NTHi are less prone to cause severe infections than Hib in unvaccinated populations [3]. Recent studies have reported an increase in invasive

\footnotetext{
* Correspondence: fredrik.resman@med.lu.se

${ }^{2}$ Department of Translational Medicine, Medical Microbiology, Lund

University, Malmö, Sweden

Full list of author information is available at the end of the article
}

disease caused by NTHi in individuals $>60$ years of age [2] and a recent review has suggested increased surveillance and typing activities of NTHi [4]. Beta-lactam resistance in $H$. influenzae is caused either by the production of beta-lactamases or by amino acid substitutions in the penicillin binding protein (PBP) 3 resulting in decreased affinity for penicillins and cephalosporins [5]. The amino acid substitution pattern can be used to divide these isolates into three different groups [6]. Outbreaks of infections caused by NTHi in long-term care facilities (LTCF) for elderly are rare and have only been reported occasionally $[7,8]$. 


\section{Outbreak description}

On October $5^{\text {th }}, 2011$, three residents (all aged 80 years or older) from an LTCF were referred to the hospital of Karlskrona, Sweden, with fever, cough and respiratory distress. Due to the suspicion of an outbreak of a communicable respiratory tract pathogen, the general practitioner (GP) responsible for the LTCF contacted the County medical officer for Communicable Disease Control (CCDC). Patients were tested for influenza virus with polymerase chain reaction (PCR) and urinary antigen test for Legionella pneumophila serogroup 1. Samples from the nasopharynx, urine and blood were obtained for bacterial culture. All patients received benzylpenicillin (PcG) intravenously according to national guidelines. Due to suspicion of influenza, oseltamivir was administered to the hospitalized as well as the eight non-infected residents at the LTCF. Both influenza and Legionella tests were negative and oseltamivir was discontinued two days later.

The LTCF consisted of twelve separate apartments occupied by 11 residents. The staff generally wore protective clothing and had access to alcohol-based hand sanitizers. On October $5^{\text {th }}$, several staff members also had respiratory symptoms and a few were on sick-leave. Four staff members with mild respiratory tract symptoms consulted the Dept. of Infectious diseases on October $6^{\text {th }}$, encouraged by the CCDC, and were in addition to nasopharyngeal cultures sampled for influenza virus A and B $(n=3)$, Mycoplasma pneumoniae $(n=4)$ and Respiratory Syncytial virus $(n=3)$. Two of the hospitalized residents were diagnosed with pneumonia as confirmed by X-ray, while the third was considered as a bacterial bronchitis. On October $8^{\text {th }}$, nasopharyngeal cultures from all three hospitalized patients grew a beta-lactamase negative $H$. influenzae with positive screening for Beta-lactam non-susceptibility and resistant to co-trimoxazole (SXT). The isolates were susceptible to tetracycline (TET) and ciprofloxacin (CIP). The isolates were further characterized with MIC testing for ampicillin (AMP) and cefotaxime (CTX), and sent to the Swedish Institute for Communicable Disease Control (SMI) for capsular typing. On October $9^{\text {th }}$, a female resident without obvious symptoms of infection died at the LTCF. The autopsy showed that the cause of death was an extensive pneumonia, and an isolate obtained from the pulmonary tissue revealed a beta-lactamase negative $\mathrm{Hi}$ with an identical susceptibility pattern as the hospitalized residents. On October $10^{\text {th }}$, one more resident from the same LTCF was admitted to the hospital with pneumonia and was treated with doxycycline at admission. The nasopharyngeal culture yielded a $\mathrm{Hi}$ isolate with the same antibiogram as the previously hospitalized patients. A summary of the clinical information available for the four hospitalized residents is presented in Table 1.

Several actions were taken by the CCDC three days after the initiation of the outbreak. Regular meetings were arranged with all responsible professionals according to the local epidemic plan. The hygiene routines at the LTCF were surveyed by the infection control team. It was agreed that there was an outbreak of an NTHi with an unusual susceptibility pattern, seemingly high virulence and ability to spread. No new residents were admitted to the LTCF until the spread was under control. Education was given regarding good hygienic routines and cohorting of staff was introduced.

\section{Methods}

\section{Patient characteristics}

A retrospective chart review was performed for the four hospitalized patients. Disease severity was evaluated using the CRB-65-system (confusion-respiratory rateblood pressure-age $>65$ years) [9].

\section{Microbiological methods}

Nasopharyngeal swabs (Copan, Brescia, Italy) were obtained from 60 individuals, as part of standard care: 10 residents (6 with/4 without symptoms), 49 staff members (7 with/42 without symptoms), and one relative to a staff member presenting with symptoms of upper respiratory tract infection. An additional sample was obtained from pulmonary tissue of the resident that died. Nasopharyngeal swabs were cultured according to standard methodology. In brief nasopharyngeal swabs were cultured on Blood agar incubated in anaerobic environment and Hematin agar incubated in $5 \% \mathrm{CO}_{2}$. Olandomycin-discs $(50 \mu \mathrm{g})$ were used to improve the detection of $H$. influenzae. Species identification was performed using MALDI-TOF (Microflex and Biotyper 3.0, Bruker Daltonics, Billerica, MA) with standard parameters. Susceptibility testing was performed according to EUCAST.

In October 2011 all $H$. influenzae isolates isolated at the Dept. of Clinical Microbiology, Karlskrona were subjected to beta-lactam resistance screening using the PCG 1 unit disc as recommended by EUCAST. Briefly, isolates displaying a zone of PCG $\leq 12 \mathrm{~mm}$ were further analysed with Cefinase disk (Biomerieux, Solna, Sweden) to detect beta-lactamase production. Beta-lactamase negative isolates were further tested with Etest (Biomerieux) on MH-F agar to evaluate the susceptibility to AMP and CTX. In retrospect 14 isolates in this study were tested with Etest for susceptibility to cefuroxime (CXM). All isolates were also tested for susceptibility to TET, SXT, nalidixic acid (NAL), cefaclor (CKL), and ampicillin/ clavulanic acid (ACL) using disk diffusion on MH-F agar according to EUCAST methodology (www.eucast.org). Finally, the total number of nasopharyngeal swabs analyzed during October 2009-2011 was retrieved from the Laboratory Information System. 


\begin{tabular}{|c|c|c|c|c|c|c|c|c|c|c|c|}
\hline Resident & Gender & $\begin{array}{l}\text { Resp. rate } \\
\text { (min) }\end{array}$ & $\begin{array}{l}\text { CRP } \\
(\mathrm{mg} / \mathrm{L})\end{array}$ & $\begin{array}{l}\text { Saturation } \\
(\%)\end{array}$ & $\begin{array}{l}\text { Temp. }\left(^{\circ}\right. \\
\text { C) }\end{array}$ & $\begin{array}{l}\text { Blood pressure } \\
(\mathrm{mmHg})\end{array}$ & $\begin{array}{l}\text { Lactate } \\
(\mathrm{mmol} / \mathrm{L})\end{array}$ & $\begin{array}{l}\text { CRB-65 } \\
\text { (score 0-4) }\end{array}$ & X-ray & $\begin{array}{l}\text { Hospital stay } \\
\text { (days) }\end{array}$ & Comorbidities \\
\hline 1 & $\mathrm{~F}$ & 30 & 302 & 80 & 37,4 & $190 / 105$ & 2.4 & 2 & $\begin{array}{l}\text { Cardiac incompensation, } \\
\text { pleural fluid }\end{array}$ & 5 & Asthma \\
\hline 2 & M & 46 & 380 & 87 & 39,2 & $87 / 58$ & 3.1 & 3 & $\begin{array}{l}\text { Infective infiltrates in } \\
\text { both lungs }\end{array}$ & 5 & Hypothyreosis \\
\hline 3 & $\mathrm{~F}$ & 28 & 269 & 81 & 37,4 & $130 / 60$ & 2 & 1 & $\begin{array}{l}\text { Infective infiltrate in } \\
\text { right lung }\end{array}$ & 4 & $\begin{array}{l}\text { Asthma, Diabetes mellitus, } \\
\text { Renal failure, Stroke, Vascular } \\
\text { dementia }\end{array}$ \\
\hline 4 & $\mathrm{~F}$ & 28 & 309 & 88 & 37,2 & $118 / 58$ & 0.9 & 1 & $\begin{array}{l}\text { Infective infiltrates in the right } \\
\text { lung }\end{array}$ & 7 & - \\
\hline
\end{tabular}




\section{Molecular typing and phylogenetic analyses}

The ftsI gene was analysed as published by Skaare et al. [10]. Consensus and protein sequences were generated using BioEdit 7.0.9.0. The available outbreak isolates as well as 31 controls (21 invasive Swedish NTHi cases [11] and 10 randomly selected Swedish nasopharyngeal isolates were sequence-typed using PCR primers and conditions according to the $H$. influenzae protocol (http://haemophilus.mlst.net/). Sequences were trimmed manually, concatenated, aligned and edited using ClustalX [12]. A best-fitting nucleotide substitution model was estimated using jModeltest 0.1.9 [13]. A Maximum likelihood tree was constructed in MEGA5.05 [14] using the General Time Reversible model including invariable sites and rate variation $(G T R+I+G)$. Support for internal branches was obtained by 1000 bootstrap replicates. The resulting phylogenetic tree was visualized using FigTree v.1.3.1 (http://tree.bio.ed.ac.uk/software/figtree).

\section{Ethics statement}

The study was performed in accordance with approval by The regional ethic review board in Lund, Sweden (2014/533).

\section{Results}

In total 15 individuals were positive for a beta-lactamase negative $H$. influenzae resistant to PCG and STX. Fourteen of these isolates were from nasopharynx (7 residents, 6 staff members, one partner to a staff member) and one from lung tissue (post-mortem culture). All isolates were susceptible to NAL and TET and displayed MIC values for AMP and CTX near the clinical breakpoints while CXM MIC was $16 \mathrm{mg} / \mathrm{L}$. The MIC for AMP were 1 or $2 \mathrm{mg} / \mathrm{L}$ (current EUCAST breakpoints $\mathrm{S} \leq 2 \mathrm{mg} / \mathrm{L}, \mathrm{R}>2 \mathrm{mg} / \mathrm{L}$ ) and for CTX 0.125 or $0.25 \mathrm{mg} / \mathrm{L}(\mathrm{S} \leq 0125 \mathrm{mg} / \mathrm{L}, \mathrm{R}>$ $0.125 \mathrm{mg} / \mathrm{L}$ ). The reported result for ACL was inferred from the MIC-value from AMP. Isolates with this particular susceptibility pattern were not observed in any other clinical samples in October 2011 in County Blekinge. The three isolates sent to the Swedish CDC for capsule typing by PCR were determined as NTHi.

Fourteen of the 15 NTHi isolates with the specific susceptibility pattern were saved and available for further analysis. Six isolates were from residents, 7 from staff members and one from a relative to a staff member. All isolates displayed the same ftsl-gene sequence with a corresponding amino acid motif of Asn526Lys (genovar IIb [14]), and a homogenous MLST-pattern where all but one outbreak isolate were subtyped to be ST 14 . Interestingly, the isolates clustered with invasive NTHi isolates from other parts of Sweden (Fig. 1).

In October 2011, 159 nasopharyngeal cultures in total were analyzed in County Blekinge. The corresponding figures for 2009 and 2010 were 94 and 113, respectively.
The proportion of $H$. influenzae during the same 3-year period (2009-2011) was $7 \%, 10 \%$ and $13 \%$. The resistance to CTX has historically in this area been very low (single isolates per year) and in samples not related to the outbreak CTX-resistance was not observed during the time of the outbreak.

The first three residents admitted to hospital were administered PcG. All three experienced an initial clinical improvement. The fourth patient received doxycycline from start. All four patients admitted to the hospital and 9 of the colonized residents and staff, were treated with doxycycline. A control culture was obtained approximately three weeks later. The outbreak strain still colonized the nasopharyngeal flora in five of these individuals. None of the residents and staff required hospitalization due to respiratory tract infections during the weeks after the outbreak.

\section{Discussion}

This outbreak demonstrates the potential virulence of subtypes of non-typeable $H$. influenzae, and highlights the important roles of all parts of the healthcare system to identify and control an outbreak. The outbreak was suspected by an alert GP, who contacted the CCDC leading to adequate sampling of the suspected index patients and quick measures of control. The detection was further facilitated by the new screening method for detection of beta-lactam resistance in H. influenzae recommended by EUCAST using PcG. The testing of AMP only had in these strains probably led to false conclusions, since AMP-resistance was not readily detected but cephalosporin resistance was pronounced. There were no geographically unrelated clinical isolates displaying the specific antibiogram identified during October 2011 in County Blekinge supporting the local characteristics of the outbreak.

A high attack rate was observed as 7 of the 11 residents were colonized, four of them were hospitalized and a fifth resident died due to pneumonia. In this patient no other pathogens than the specific Hi-strain were isolated from lung tissue. Several of the staff, without underlying disease, experienced respiratory tract symptoms at the time of the outbreak and in 6 of these the outbreak strain was isolated. The pathogenicity of NTHi is substantially lower and different than that of Hib [3], but in this outbreak an NTHi strain caused severe infections supporting the notion that some lineages of NTHi have a higher virulence. MLST and ftsI-gene sequencing showed a clonal distribution of the epidemiologically related isolates, and the main ST-type was 14 . Interestingly, the outbreak isolates clustered with a recently acknowledged lineage of invasive isolates of NTHI with the same ftsI sequence from several larger regions in Sweden [11]. The ST 14 cluster has also been demonstrated as the subtype most prone to cause hospitalization among subtypes of NTHi in a recent 


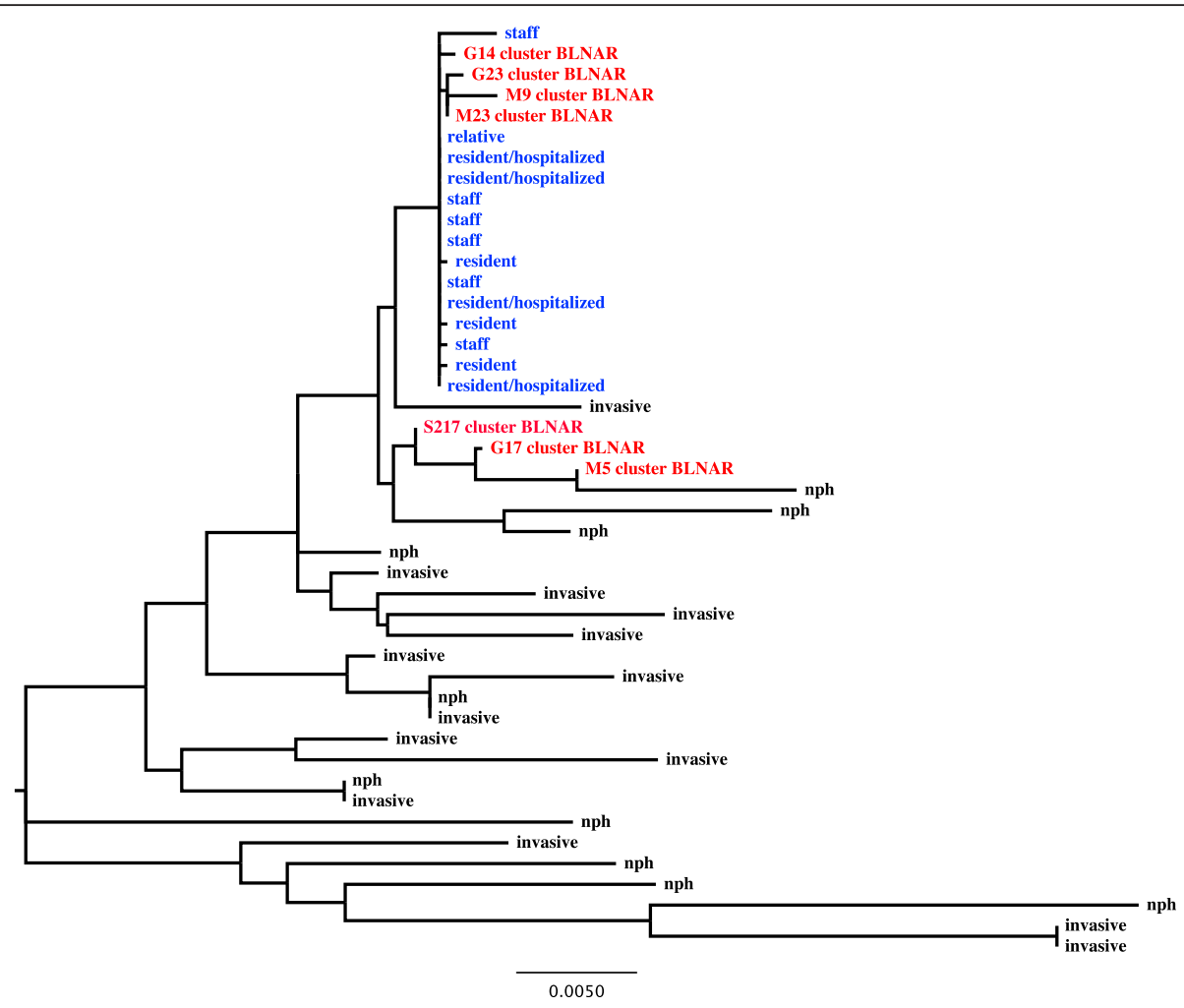

Fig. 1 A phylogenetic tree from concatenated MLST sequences of the outbreak isolates $(n=14)$ as well as of 31 control isolates. The outbreak isolates are shown in blue text. Isolates from a suggested cluster of BLNAR genotype Ilb isolates with identical PBP-3 sequences are shown in red $(n=7)$. Randomly selected controls are shown in black, where nasopharyngeal controls are denoted as "nph", and invasive control are denoted as "invasive". The tree shows a clonal clustering of the outbreak isolates, with one isolate separated from the rest. Interestingly, the outbreak isolates cluster with the suggested BLNAR clone [15]

Norwegian study [15]. In vitro studies have indicated that NTHi with specific changes in PBP3 may be more prone to invasion into the host epithelial cells through micropinocytosis [5], even though this is not likely to be related to the PBP-3 change per se [16]. It is unlikely that the isolation of the same $H$. influenzae in all cases would be coincidental, and that the outbreak was due to another microorganism. Haemophilus influenzae is not a common colonizer of the respiratory tract in healthy adults such as the staff [17], and a in a recent screening of 98 non-infected residents of a different Swedish LTCF, no resident was colonized with $H$. influenzae (own unpublished data).

According to Swedish national guidelines patients with moderate pneumonia are to be treated with i.v. PcG 1-3 $g \times 3$ (www.infektion.net). The first three residents admitted to hospital were administered PcG. Interestingly, all three experienced an initial clinical improvement despite the, from a microbiological point of view, ineffective therapy. This might be in part due to the supportive therapy, but CRP-values were also lower following three days of treatment indicating an effect on the infection. As bacterial cultures from the lower respiratory tracts were not obtained, we might have underestimated the presence of other bacterial pathogens (i.e. pneumococci) in these patients. However, a recent retrospective analysis of invasive $H$. influenzae infections suggested that patient empirically treated with PcG, had a good prognosis if the treatment was altered according to culture results, while worse outcomes were seen in patients with a definite treatment of PcG in comparison to CXM [18]. Thirteen of the 15 residents and staff that were positive for the outbreak strain were eventually treated with doxycycline. A control culture was obtained approximately three weeks later. The outbreak strain still colonized the nasopharyngeal flora in five of these individuals despite adequate doxycycline treatment. No further treatment was administered, and no further cases were seen after the actions taken by the $C C D C$, despite the fact that some staff and residents were still colonized with the outbreak strain.

\section{Conclusions}

We conclude that a limited outbreak of a beta-lactamase negative NTHi ST 14 with MIC values for AMP and 
CTX just at the clinical breakpoints occurred and was associated with severe disease. The outbreak may have been missed if ampicillin only had been used to detect BLNARs, but these strains were easily detected with the EUCAST recommended PCG $1 \mathrm{U}$ disk screen. The outbreak strain was closely related to a recently acknowledged lineage of NTHI. The particular NTHi clone (ST-14) seems to be associated with severe infections and further studies are needed to elucidate the virulence mechanisms and epidemiology of this particular clone.

\begin{abstract}
Abbreviations
ACL: amoxicillin-clavulanic acid; AMP: ampicillin; CCDC: County medical officer for Communicable Disease Control; CKL: cefaklor; CRB-65: Confusion-respiratory rate-blood pressure-age $>65$ years; CTX: cefotaxime; CXM: cefuroxime; EUCAST: European Committee on Antibiotic Susceptibility Testing; GP: general practitioner; Hi: Haemophilus influenzae; LTCF: long-term care facility; MIC: Minimum Inhibitory Concentration; MLST: Multi Locus Sequence Typing; NAL: nalidixic acid; NTHi: non typeable Haemophilus influenzae; PBP: penicillin-binding protein; PCG: benzylpenicillin; PCR: Polymerase Chain Reaction; SXT: co-trimoxazole; TET: tetracycline.
\end{abstract}

\section{Competing interests}

The authors declare that they have no competing interests.

\section{Authors' contributions}

MA and RE collected and analyzed clinical data. RE performed interventions during the outbreak. FR performed the MLST analyses and analyzed data. PD performed the PBP mutation analyses. KR and GK assisted in the analyses of data and had input on study design. MS collected microbiological data and coordinated the study design and the analyses of data. MA, FR, RE and MS drafted the manuscript with substantial input from PD, KR and GK. All authors read and approved the final version of the manuscript.

\section{Acknowledgements}

Kerstin Goos and Marie Corneliusson for technical assistance. This work was supported by a grant (to KR) from the Skåne County Council's research and development foundation.

\section{Author details \\ 'Department of Infectious Diseases, Blekinge Hospital, Karlskrona, Sweden. ${ }^{2}$ Department of Translational Medicine, Medical Microbiology, Lund University, Malmö, Sweden. ${ }^{3}$ Department of Communicable Disease Control, County Blekinge, Karlskrona, Sweden. ${ }^{4}$ Department of Clinical Microbiology, County Kronoberg, Växjö/Karlskrona, Sweden. ${ }^{5}$ Department of Medical Sciences, Division of Clinical Bacteriology, Uppsala University, Uppsala, Sweden. ${ }^{6}$ Department of Laboratory Medicine, Clinical Microbiology, Faculty of Medicine and Health, Örebro University, Örebro, Sweden.}

Received: 29 June 2015 Accepted: 12 December 2015

Published online: 23 December 2015

\section{References}

1. Murphy TF. Respiratory infections caused by non-typeable Haemophilus influenzae. Curr Opin Infect Dis. 2003;16(2):129-34. PubMed.

2. Resman F, Ristovski M, Ahl J, Forsgren A, Gilsdorf JR, Jasir A, et al. Invasive disease by Haemophilus influenzae in Sweden 1997-2009; evidence of increasing incidence and clinical burden of non-type b strains. Clin Microbiol Infect. 2010. PubMed Epub 2010/11/09. Eng.

3. Murphy TF, Faden H, Bakaletz LO, Kyd JM, Forsgren A, Campos J, et al. Nontypeable Haemophilus influenzae as a pathogen in children. Pediatr Infect Dis J. 2009;28(1):43-8. PubMed Epub 2008/12/06.

4. Van Eldere J, Slack MPE, Ladhani S, Cripps AW. Non-typeable Haemophilus influenzae, an under-recognised pathogen. Lancet Infect Dis. 2014;14:1281-92. PubMed Epub 2014 Jul 7.

5. Okabe T, Yamazaki Y, Shiotani M, Suzuki T, Shiohara M, Kasuga E, et al. An amino acid substitution in PBP-3 in Haemophilus influenzae associate with the invasion to bronchial epithelial cells. Microbiol Res. 2010;165(1):11-20. PubMed Epub 2008/04/18. eng.

6. Dabernat $H$, Delmas C, Seguy M, Pelissier R, Faucon G, Bennamani S, et al. Diversity of beta-lactam resistance-conferring amino acid substitutions in penicillin-binding protein 3 of Haemophilus influenzae. Antimicrob Agents Chemother. 2002;46(7):2208-18. Epub 2002/06/19. eng.

7. Van Dort M, Walden C, Walker ES, Reynolds SA, Levy F, Sarubbi FA. An outbreak of infections caused by non-typeable Haemophilus influenzae in an extended care facility. J Hosp Infect. 2007;66(1):59-64. PubMed.

8. Yang CJ, Chen TC, Wang CS, Wang CY, Liao LF, Chen YH, et al. Nosocomial outbreak of biotype I, multidrug-resistant, serologically non-typeable Haemophilus influenzae in a respiratory care ward in Taiwan. J Hosp Infect. 2010;74(4):406-9. PubMed.

9. Bauer TT, Ewig S, Marre R, Suttorp N, Welte T, Group CS. CRB-65 predicts death from community-acquired pneumonia. J Intern Med. 2006;260(1):93-101. PubMed.

10. Skaare D, Allum AG, Anthonisen IL, Jenkins A, Lia A, Strand L, et al. Mutant fts genes in the emergence of penicillin-binding protein-mediated beta-lactam resistance in Haemophilus influenzae in Norway. Clin Microbiol Infect. 2010; 16(8):1117-24. PubMed Epub 2009/09/10. eng.

11. Resman F, Ristovski M, Forsgren A, Kaijser B, Kronvall G, Medstrand P, et al. Increase of beta-lactam-resistant invasive Haemophilus influenzae in Sweden, 1997 to 2010. Antimicrob Agents Chemother. 2012;56(8):4408-15. Epub 2012/06/13. eng.

12. Larkin MA, Blackshields G, Brown NP, Chenna R, McGettigan PA, McWilliam H, et al. Clustal W and Clustal X version 2.0. Bioinformatics. 2007;23(21):2947-8. PubMed.

13. Posada D. jModelTest: phylogenetic model averaging. Mol Biol Evol. 2008; 25(7):1253-6. PubMed Epub 2008/04/10. eng.

14. Tamura K, Peterson D, Peterson N, Stecher G, Nei M, Kumar S. MEGA5: molecular evolutionary genetics analysis using maximum likelihood, evolutionary distance, and maximum parsimony methods. Mol Biol Evol. 2011;28(10):2731-9.

15. Skaare D, Anthonisen IL, Caugant DA, Jenkins A, Steinbakk M, Strand L, et al. Multilocus sequence typing and ftsl sequencing: a powerful tool for surveillance of penicillin-binding protein 3-mediated beta-lactam resistance in nontypeable Haemophilus influenzae. BMC Microbiol. 2014;14:131.

16. Atkins NA, Kunde DA, Zosky G, Tristram SG. Genotypically defined betalactamase-negative ampicillin-resistant isolates of non-typable Haemophilus influenzae are associated with increased invasion of bronchial epithelial cells in vitro. J Med Microbiol. 2014;63(Pt 10):1400-3. PubMed.

17. Gunnarsson RK, Holm SE, Soderstrom M. The prevalence of potential pathogenic bacteria in nasopharyngeal samples from healthy children and adults. Scand J Prim Health Care. 1998;16(1):13-7. PubMed.

18. Thonnings S, Ostergaard C. Treatment of Haemophilus bacteremia with benzylpenicillin is associated with increased (30-day) mortality. BMC Infect Dis. 2012;12:153.

Submit your next manuscript to BioMed Central and we will help you at every step:

- We accept pre-submission inquiries

- Our selector tool helps you to find the most relevant journal

- We provide round the clock customer support

- Convenient online submission

- Thorough peer review

- Inclusion in PubMed and all major indexing services

- Maximum visibility for your research

Submit your manuscript at www.biomedcentral.com/submit 